\title{
CARACTERIZAÇÃO ESPECTROSCÓPICA E FISICO- QUÍMICA DE FILMES DE BLENDAS DE AMIDO E CARBOXIMETILCELULOSE.
}

\author{
C. H. FERREIRA ${ }^{1}$, E. F. PASSOS ${ }^{1}$, P. T. MARQUES ${ }^{1}$ \\ ${ }^{1}$ Universidade Tecnológica Federal do Paraná - Pato Branco - Departamento de Química \\ E-mail para contato: carlisehannelferreira@gmail.com \\ elthonpassos.quimica@gmail.com
}

\begin{abstract}
RESUMO - Os polímeros são umas das classes de materiais mais versáteis, tendo uma grande aplicação dentro dos mais diversos ramos da indústria. Sendo assim, foram preparados filmes de amido e $\mathrm{CMC}$ com e sem adição de óxido de magnésio $(\mathrm{MgO})$, e realizados estudos de caracterização espectroscópica no infravermelho e testes de solubilidade para analisar as características das blendas formadas. As análises espectroscópicas mostram que houve pouca alteração nas bandas de interesse, em comparação com os espectros dos filmes puros. A adição de $(\mathrm{MgO})$ não exerceu influência nas blendas. A análise visual dos filmes não indica a presença de fase nos mesmos. No teste de solubilidade, o filme contendo amido (1g) e $\mathrm{MgO}$ em concentração de $0,002 \mathrm{~g}$ apresenta menor porcentagem de material seco solubilizado (\%MS).
\end{abstract}

\section{INTRODUÇÃO}

De acordo com Canevarolo (2006), os polímeros são macromoléculas, formadas pela repetição de unidades menores denominadas monômeros. De acordo com suas características, os polímeros podem ser classificados em diversos grupos, porém, de maneira geral, estes são divididos em dois grupos, relacionados à sua origem, sendo eles, os polímeros naturais aqueles encontrados na natureza - e os sintéticos - produzidos em laboratório. (MANO; MENDES, 1999).

A celulose, e o amido estão inclusos na classe dos polímeros naturais, e são compostos abundantes na natureza. Devido a características de grande importância como biodegradabilidade e, por serem recursos renováveis, ambos os compostos podem ser empregados nas mais diversas aplicações e estão entre os principais polissacarídeos de interesse industrial.

Como forma de obtenção de materiais com propriedades melhoradas, como, por exemplo, a resistência mecânica e térmica, as blendas poliméricas surgem como uma boa alternativa para as diversas necessidades apresentadas pelas indústrias, tendo, assim como os polímeros puros, sua aplicação destinada aos mais variados setores, deste a indústria de embalagens à farmacêutica (TONHI; PLEPLIS, 2002). 
O objetivo deste trabalho foi produzir filmes de amido de mandioca e carboximetilcelulose (CMC), na presença e ausência de óxido de magnésio, em diferentes proporções. Estes materiais foram caracterizados por espectroscopia, solubilidade em água e miscibilidade aparente.

\section{MATERIAIS E MÉTODOS}

\subsection{Preparo dos filmes}

Os filmes foram preparados dissolvendo-se as quantidades de amido e CMC separadamente em béqueres contendo $100 \mathrm{~mL}$ de água destilada, de acordo com as proporções mostradas na tabela abaixo (Tabela 1). A solução contendo amido foi aquecida até $80^{\circ} \mathrm{C}$ por um período de 30 minutos sob agitação magnética. A CMC foi preparada deixando a solução sob agitação por 1 hora sem aquecimento. As blendas de amido e CMC foram preparadas com e sem o $\mathrm{MgO}$ e as soluções foram deixadas sob agitação por $1 \mathrm{~h}$. As proporções trabalhadas são apresentadas na tabela 1. As soluções filmogênicas foram adicionadas em placas de pétri e deixadas secar em estufa com circulação de ar sob temperatura de $40^{\circ} \mathrm{C}$ por $48 \mathrm{~h}$. Os filmes foram preparados utilizando-se fécula de mandioca (amido) obtida em comércio local, carboximetilcelulose - CMC (Synth) e óxido de Magnésio $(\mathrm{MgO})$.

Tabela 1 - Proporções dos filmes poliméricos

\begin{tabular}{cccccc}
\hline Filme & $\begin{array}{c}\text { CMC } \\
\mathbf{0 , 5 g}\end{array}$ & $\begin{array}{c}\text { Amido } \\
\mathbf{1 , 0 g}\end{array}$ & $\begin{array}{c}\text { Amido } \\
\mathbf{2 , 5 g}\end{array}$ & $\begin{array}{c}\text { MgO } \\
\mathbf{0 , 0 0 2} \mathbf{g}\end{array}$ & $\begin{array}{c}\text { MgO } \\
\mathbf{0 , 0 0 4 g}\end{array}$ \\
\hline $\mathbf{1}$ & $\checkmark$ & & & & \\
\hline $\mathbf{2}$ & & $\checkmark$ & & & \\
\hline $\mathbf{3}$ & & & $\checkmark$ & & \\
\hline $\mathbf{4}$ & $\checkmark$ & $\checkmark$ & & & \\
\hline $\mathbf{5}$ & $\checkmark$ & $\checkmark$ & & $\checkmark$ & $\checkmark$ \\
\hline $\mathbf{6}$ & $\checkmark$ & $\checkmark$ & & & \\
\hline $\mathbf{7}$ & $\checkmark$ & & $\checkmark$ & & $\checkmark$ \\
\hline $\mathbf{8}$ & $\checkmark$ & & $\checkmark$ & $\checkmark$ & $\checkmark$ \\
\hline $\mathbf{9}$ & $\checkmark$ & & $\checkmark$ & & $\checkmark$ \\
\hline
\end{tabular}

\subsection{Análise Espectroscópica}

Essas análises foram realizadas na região do infravermelho médio com transformada de Fourier, em um equipamento modelo Perkin-Elmer equipado com acessório UATR e 
cristal de carbono (diamante). A área dos filmes para a análise foi de 1,0x1,0 cm. As amostras foram submetidas a uma alta pressão a fim de se obter um espectro de melhor qualidade. A faixa espectral considerada foi de 4000 a $400 \mathrm{~cm}^{-1}$, realizando 16 varreduras e utilizando uma resolução de $4 \mathrm{~cm}^{-1}$.

\subsection{Solubilidade em água e miscibilidade}

A miscibilidade dos filmes foi analisada a olho nu, observando-os contra a luz. A solubilidade em água foi determinada, retirando-se uma amostra de $4 \mathrm{~cm}^{2}$, as quais foram pesadas e submersas em $5 \mathrm{~mL}$ de água destilada. O sistema foi deixado em repouso durante $24 \mathrm{~h}$ em temperatura ambiente. Após este período as amostras foram secas em estufa com circulação de ar $\left(105^{\circ} \mathrm{C}-12 \mathrm{~h}\right)$. Este método foi adaptado a partir da metodologia utilizada por Tongdeesoontorn et. al. (2011). A solubilidade foi calculada utilizando-se a equação 1:

$\% M S=\left(\frac{P I-P F}{P I}\right) \times 100$,

onde: \%MS é a porcentagem de material seco solubilizado; PI é o peso inicial do material seco; e PF é o peso final do material seco não solubilizado.

\subsection{Espessura dos filmes}

A medida da espessura dos filmes foi realizada com micrômetro digital Insize (0$25 \mathrm{~mm} / 0-1$ "), efetuando medições em 4 regiões distintas dos filmes e considerando o valor médio entre elas.

\section{RESULTADOS E DISCUSSÃO}

\subsection{Análise dos espectros de infravermelho}

Os espectros obtidos para as amostras de filmes puros de amido são mostrados nas figuras 1(b) e 2(b). Observa-se uma banda intensa, em $3301 \mathrm{~cm}^{-1}$ devido ao estiramento do grupo $\mathrm{OH}$. Em $2923 \mathrm{~cm}^{-1}$ observa-se uma banda que é atribuída ao estiramento do grupo C-H. Também na região entre 1646 e $1649 \mathrm{~cm}^{-1}$ os espectros mostram uma banda que indica a presença de água ligada a estrutura do polímero, enquanto que em $1411 \mathrm{~cm}^{-1}$ e $1365 \mathrm{~cm}^{-1}$ há bandas atribuídas ao dobramento dos grupos $\mathrm{CH}_{2}$ e C-OH, respectivamente. O estiramento do grupo C-O-C pode ser observado na região de $1144 \mathrm{~cm}^{-1}$. Esses resultados corroboram com os observados por Tongdeesoontorn et. al. (2011). Segundo Vicentini (2003) as bandas que ocorrem na região de $1000 \mathrm{~cm}^{-1}$ estão relacionadas a estrutura cristalina do amido, assim, as bandas observadas nas Figuras 1 e 2 do presente trabalho, em $994 \mathrm{~cm}^{-1}$ e $1013 \mathrm{~cm}^{-1}$, sugerem que o filme possui estrutura amorfa, seguindo também em concordância com o mencionado por VanSoest et. al. (1995) apud Henrique et. al. (2007). 
Os espectros dos filmes puros de CMC (figuras 1a e 2a) mostraram, também em 3301 $\mathrm{cm}^{-1}$, uma banda referente à vibração de estiramento do grupo $\mathrm{OH}$, enquanto que a banda atribuída ao estiramento $\mathrm{CH}$ ocorreu na região entre $2886-2917 \mathrm{~cm}^{-1}$. Em $1411 \mathrm{~cm}^{-1}$ e 1319 $\mathrm{cm}^{-1}$ as bandas com picos intensos referem-se à vibração de dobramento dos grupos $\mathrm{CH}_{2} \mathrm{e}$ $\mathrm{OH}$, respectivamente. Em torno de $1052 \mathrm{~cm}^{1}$ a banda observada deve-se, provavelmente, ao estiramento $\mathrm{CH}-\mathrm{O}-\mathrm{CH}_{2}$. O espectro mostrou também, uma banda em $1587 \mathrm{~cm}^{-1}$ que é atribuída à vibração antissimétrica do grupo $\mathrm{COO}^{-}$.

As blendas de Amido e CMC (sem MgO) analisadas mostram um pequeno deslocamento da banda referente ao estiramento do grupo $\mathrm{OH}$, observada em $3295 \mathrm{~cm}^{-1}$. Também é possível observar um deslocamento da banda referente ao dobramento de $\mathrm{C}-\mathrm{OH}$ do amido, que ocorre em $1326 \mathrm{~cm}^{-1}$. A banda atribuída à presença de água ligada a estrutura do polímero, observada anteriormente na região de $1646-1649 \mathrm{~cm}^{-1}$ praticamente não é observada com a adição de CMC. Os resultados obtidos para a CMC também seguem em concordância com os valores observados por Tongdeesoontorn et. al. (2011).

A análise espectroscópica dos materiais indicou que a adição de óxido de magnésio não exerceu efeito sobre a intensidade ou deslocamento das bandas. Os espectros obtidos e analisados das amostras puras e blendas produzidas, são mostrados nas figuras 1 e 2 a seguir:

Figura 1 - FTIR: (a) CMC pura - controle; (b) amido puro (1,0g) - controle; (c) adição de $\mathrm{CMC}-0,5 \mathrm{~g}$; (d) adição de $\mathrm{MgO}$ - 0,002g; (e) adição de $\mathrm{MgO}$ - 0,004g.

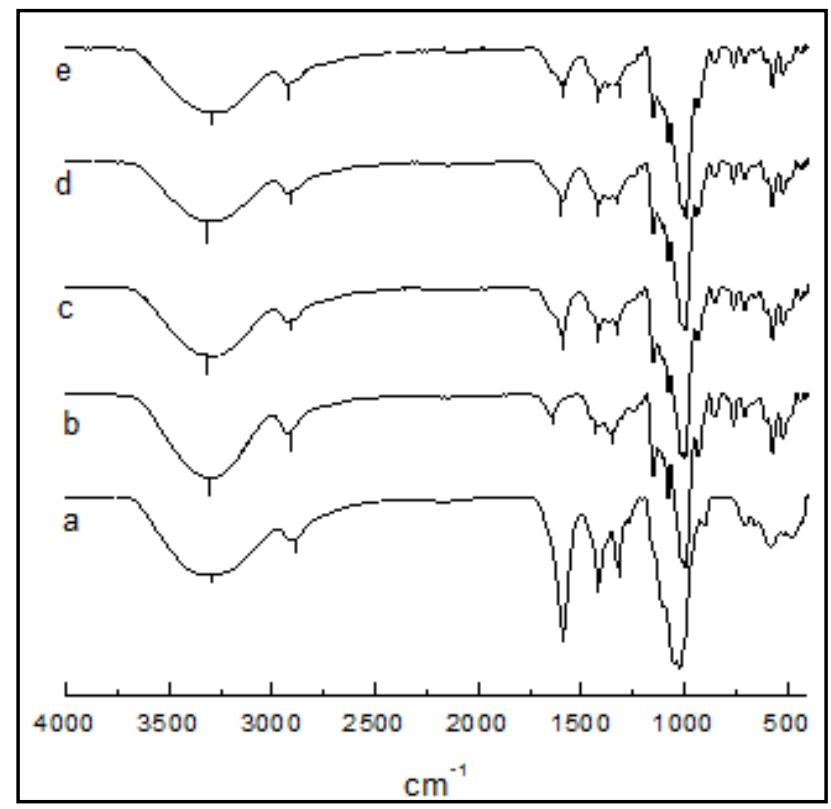

Figura 2 - FTIR: (a) CMC pura - controle; (b) amido puro (2,5g) - controle; (c) adição de CMC - 0,5g; (d) adição de $\mathrm{MgO}-0,002 \mathrm{~g}$; (e) adição de $\mathrm{MgO}-0,004 \mathrm{~g}$ 

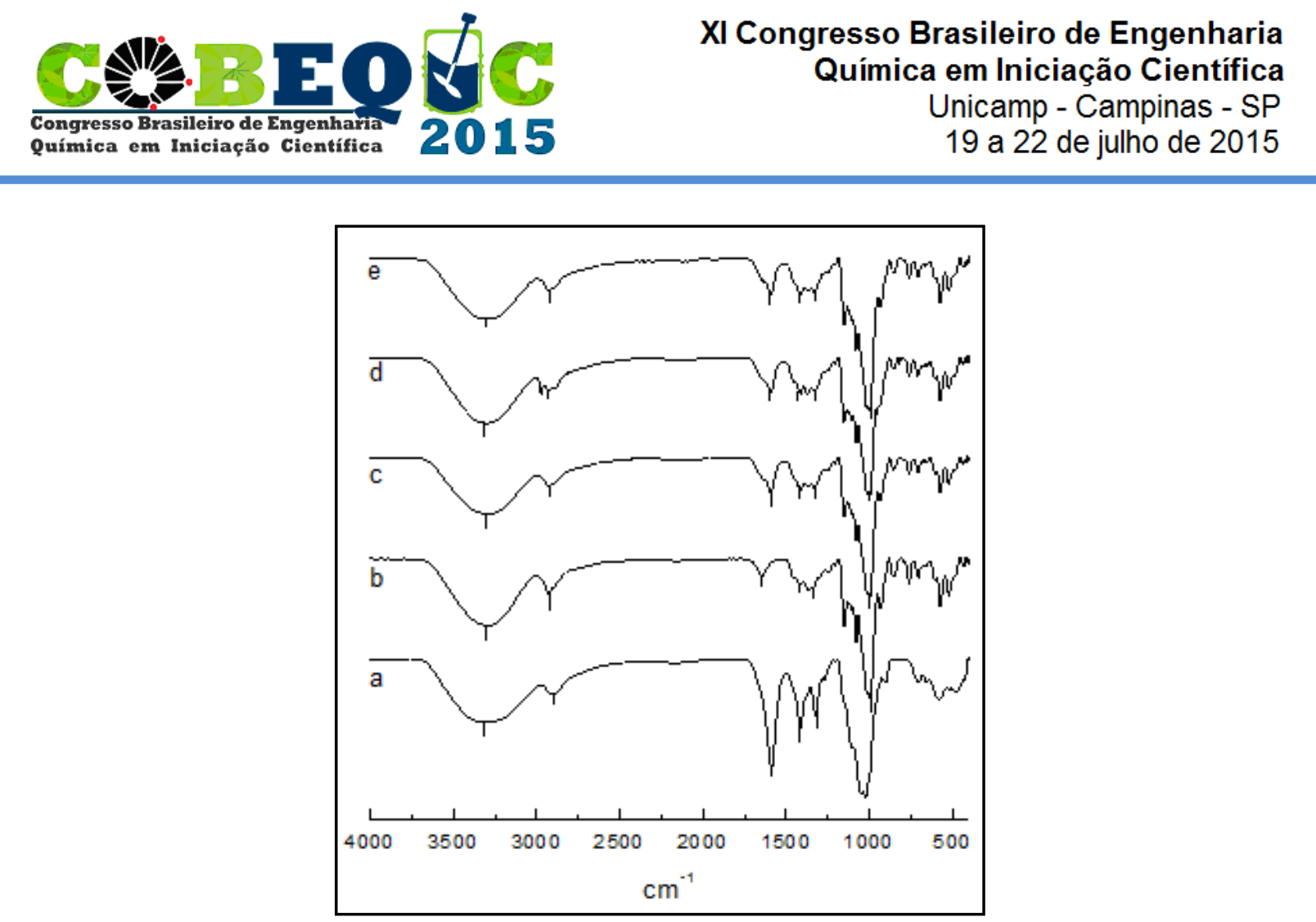

\subsection{Análise de solubilidade em água e miscibilidade}

A espessura média obtida das amostras analisadas foi de $0,069 \mathrm{~mm}$, com os valores variando entre $0,048 \mathrm{~mm}$ e $0,105 \mathrm{~mm}$. Os valores de $\% \mathrm{MS}$ obtidos para cada amostra são mostrados na tabela a seguir (Tabela 2). A numeração das amostras segue o padrão aplicado anteriormente na tabela 1 .

Tabela 2 - Valores de \%MS - porcentagem de material seco solubilizado das amostras e espessura média dos filmes $\left(\mathrm{E}_{\mathrm{mf}}\right)$ em milímetros $(\mathrm{mm})$

\begin{tabular}{cccccccccc}
\hline Filmes & $\mathbf{1}$ & $\mathbf{2}$ & $\mathbf{3}$ & $\mathbf{4}$ & $\mathbf{5}$ & $\mathbf{6}$ & $\mathbf{7}$ & $\mathbf{8}$ & $\mathbf{9}$ \\
\hline $\mathbf{\%} \mathbf{M S}$ & 97,0 & 0,5 & 13,0 & 85,7 & 8,8 & 26,2 & 13,4 & 13,9 & 18,5 \\
\hline $\mathbf{E}_{\mathbf{m f}}$ & 0,054 & 0,061 & 0,105 & 0,048 & 0,068 & 0,051 & 0,086 & 0,063 & 0,085 \\
\hline
\end{tabular}

Observa-se que o filme com maior porcentagem de solubilidade foi aquele composto apenas por CMC (filme 1), sendo que o que obteve melhor desempenho no teste aplicado foi o filme puro de amido $1 \mathrm{~g}$ (filme 2). As blendas produzidas com maior teor de amido $(2,5 \mathrm{~g}$ ) apresentaram valores próximos de solubilidade, variando entre $13,4 \%$ e $18,5 \%$, enquanto que às blendas com menor teor de amido $(1,0 \mathrm{~g})$ mostraram valores mais dispersos, sendo que $\mathrm{o}$ melhor desempenho foi observado no filme contendo óxido de magnésio em menor concentração.

Em relação à miscibilidade dos filmes produzidos, as amostras apresentaram boa homogeneidade, sendo que, em alguns casos, por conta do ambiente de secagem não possuir 


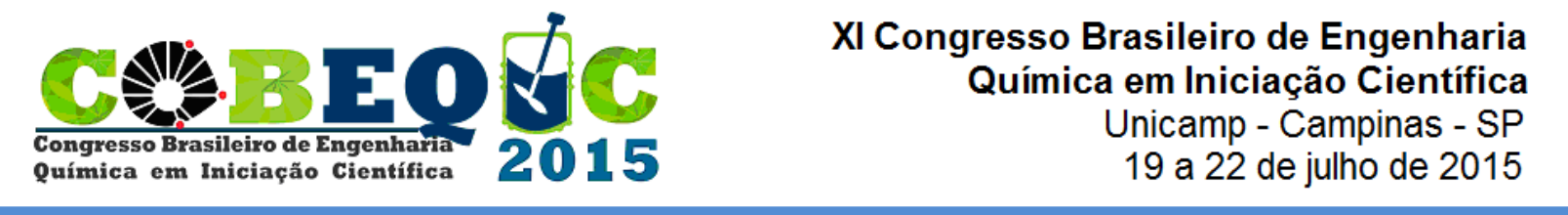

um controle rigoroso de humidade, os filmes apresentaram pequenas áreas de opacidade, atribuída ao fato de a secagem não ocorrer uniformemente nas placas.

\section{CONSIDERAÇÕES FINAIS}

Os filmes preparados não apresentaram formação de fase aparente. A caracterização espectroscópica realizada indicou que a adição de $\mathrm{MgO}$ não exerceu influência nos filmes, com relação a miscibilidade aparente e caracterização espectroscópica. Nas misturas sem a adição do óxido, os espectros indicam pequenas mudanças que seguem em concordância com valores observados em literatura, no entanto, quando submetidos a teste de solubilidade, a blenda que obteve um melhor desempenho foi aquela em que havia $\mathrm{MgO}$ em menor concentração.

\section{REFERÊNCIAS}

CANEVAROLO JR., S. V., Ciência dos Polímeros, 2a. Edição, Artliber Editora, São Paulo, 2006.

HENRIQUE, C. M.; MARNEY, P. C.; DUPUY, N. Análise de cor, transparência e infravermelho dos filmes de fécula modificada de mandioca. Agronomia Tropical. v. 57, p. 25-30, 2007.

MANO, E. B. \& MENDES, L. C; Introdução a polímeros, 2a ed., Edgard Blücher Ltda: São Paulo, 1999.

TONGDEESOONTORN, W.; MAUER, L. J.; WONGRUONG, S.; SRIBURI, P.; RACHTANAPUN, P.; Effect of carboxymethyl celulose concentration on physical properties of biodegradable cassava starch-based films. Chemistry Central Journal. 5:6, 2011.

PLEPIS, A. M. de G.; TONHI, E. Obtenção e caracterização de blendas Colágeno-Quitosana. Química Nova. v. 25, p. 943-948, 2002.

VICENTINI, N. M. Elaboração e caracterização de filmes comestíveis à base de fécula de mandioca para uso em pós-colheita. 2003, Tese; UNESP. 